\title{
Pharmacists out of this world
}

\author{
Vincent Nguyen
}

WITH THE RAPID ADVANCEMENTS OF TECHNOLOGY, there is increasing talk about space travel, particularly exploration of planets further than our moon. Elon Musk has been at the forefront of human colonization on Mars. His company, SpaceX, is currently working on a spacecraft that will be fast and efficient enough to travel to the red planet. It has been said that the spacecraft could also be used for travel on Earth, which would allow future individuals to travel anywhere on the planet in under an hour. ${ }^{1}$ Musk hopes that the first launch will be in 2022. ${ }^{1}$ If the launch is successful, then 4 spacecrafts will be going to Mars in 2024, 2 of which will have crews in them. ${ }^{1}$ This begs the question: will pharmacists be involved in the colonization of Mars?

\section{Pharmacists at NASA}

Pharmacists have been working with the National Aeronautics and Space Agency (NASA) since the beginning of the century, so pharmacist involvement in the colonization of Mars is inevitable. Tina Bayuse was the first pharmacist to work for NASA and now she is leading a team of 4 at the Johnson Space Center (JSC) in Houston. ${ }^{2}$ Bayuse was always interested in space travel as a child, but it was attending a presentation about how drugs may not function the same in space as they do on Earth that really sparked her interest in the field and encouraged her to collaborate with NASA. ${ }^{2}$ She obtained a pharmacy rotation in JSC's pharmacology lab and worked on drug monographs for the space shuttle medical kits. ${ }^{2}$ This led to the creation of the first pharmacist position within NASA.

At NASA, pharmacists mainly focus on preparing "convenience" and "contingency" medical kits for astronauts at the International Space Station. ${ }^{2}$ The main difference between the 2 kits is that the convenience kit contains medicines that one would usually take on a trip, while the contingency kit is stocked for emergencies and contains items like antibiotics and cardiac life support. Pharmacists decide what goes into the kit and then pack them into the flight kits.

The way that medications in space work may be more than a little different than the way they work on Earth. Pharmacists at NASA have to choose medication based on volume and mass, which is sometimes problematic, with some formulations including soluble powders or liquids. ${ }^{2}$ Additionally, pharmacists must think about how astronauts take their medications in space. For instance, using an injectable in an emergency is out of the question if they're wearing a space suit. Lastly, there are a lot of unknowns in space, including how the medication will react to radiation outside Earth's orbit. This is where the role of the pharmacist will be a crucial component of space travel.

\section{Future involvement}

With humans travelling to Mars as early as 2024, it is evident that more pharmacists will be needed on the NASA team. Travelling to Mars will be far different than travelling to the International Space Station-one trip is leaving Earth almost indefinitely for an unknown land, while the other allows humans to return relatively easily. Preparing medical kits for Mars will have to factor in the long-term potential risks, which may prove to be a lot more difficult than short-term risks. Pharmacists will need to think about diseases or disorders that may arise later in life and prepare adequately for those. Additionally, pharmacists will need to consider how space travel affects a person and his or her vulnerability to certain diseases or side effects to medications as well.

Pharmacists are presently and will continue to be crucial to space travel, especially when colonization on Mars occurs. Once that happens, there will potentially be a large influx of humans 
wanting to travel to Mars, not just astronauts. of humans to Mars, and eventually, pharmacists Pharmacists will be there to ensure the safe travel $\quad$ will be out of this world and on Mars as well.

From the Leslie Dan Faculty of Pharmacy, University of Toronto, Toronto, Ontario. Contact vn.nguyen@mail.utoronto.ca or vn11nguyen@gmail.com.

Author Contributions: Vincent Nguyen is the sole author of this article and is responsible for its content.

Declaration of Conflicting Interests: The author declared no potential conflicts of interest with respect to the research, authorship and/or publication of this article.

Funding: The author received no financial support for the research, authorship and/or publication of this article.

\section{References}

1. Slezak M, Solon O. Elon Musk: SpaceX can colonise Mars and build moon base. The Guardian 29 Sept 2017. Available: https://www.theguardian.com/technology/2017/sep/29/ elon-musk-spacex-can-colonise-mars-and-build-base-onoon (accessed Oct. 13, 2017).
2. Page E. How Tina Bayuse became the first pharmacist at NASA. Pharmaceutical Journal 5 Feb 2016. Available: www.pharmaceutical-journal.com/careers/careerprofile/how-tina-bayuse-became-the-first-pharmacist-atnasa/20200530.article (accessed Oct. 13, 2017). 